\title{
Identification of methylation sites and signature genes with prognostic value for luminal breast cancer
}

Bin Xiao ${ }^{1 \dagger}$, Lidan Chen ${ }^{1+}$, Yongli Ke², Jianfeng Hang ${ }^{1}$, Ling Cao ${ }^{1}$, Rong Zhang ${ }^{1}$, Weiyun Zhang ${ }^{1}$, Yang Liao ${ }^{1}$, Yang Gao ${ }^{3,4,5}$, Jianyun Chen', Li Li ${ }^{2}$, Wenbo Hao ${ }^{3,4,5}$, Zhaohui Sun $^{1 *}$ and Linhai $\mathrm{Li}^{1 *}$ (D)

\begin{abstract}
Background: Robust and precise molecular prognostic predictors for luminal breast cancer are required. This study aimed to identify key methylation sites in luminal breast cancer, as well as precise molecular tools for predicting prognosis.

Methods: We compared methylation levels of normal and luminal breast cancer samples from The Cancer Genome Atlas dataset. The relationships among differentially methylated sites, corresponding mRNA expression levels and prognosis were further analysed. Differentially expressed genes in normal and cancerous samples were analysed, followed by the identification of prognostic signature genes. Samples were divided into low- and high-risk groups based on the signature genes. Prognoses of low- and high-risk groups were compared. The Gene Expression Omnibus dataset were used to validate signature genes for prognosis prediction. Prognosis of low- and high-risk groups in Luminal A and Luminal B samples from the TCGA and the Metabric cohort dataset were analyzed. We also analysed the correlation between clinical features of low- and high- risk groups as well as their differences in gene expression.
\end{abstract}

Results: Fourteen methylation sites were considered to be related to luminal breast cancer prognosis because their methylation levels, mRNA expression and prognoses were closely related to each other. The methylation level of SOSTDC1 was used to divide samples into hypo- and hyper-methylation groups. We also identified an mRNA signature, comprising eight transcripts, ESCO2, PACSIN1, CDCA2, PIGR, PTN, RGMA, KLK4 and CENPA, which was used to divide samples into low- and high-risk groups. The low-risk group showed significantly better prognosis than the high-risk group. A correlation analysis revealed that the risk score was an independent prognostic factor. Low- and high- risk groups significantly correlated with the survival ratio in Luminal A samples, but not in Luminal B samples on the basis of the TCGA and the Metabric cohort dataset. Further functional annotation demonstrated that the differentially expressed genes were mainly involved in cell cycle and cancer progression.

Conclusions: We identified several key methylation sites and an mRNA signature for predicting luminal breast cancer prognosis. The signature exhibited effective and precise prediction of prognosis and may serve as a prognostic and diagnostic marker for luminal breast cancer.

Keywords: Luminal breast cancer, Methylation, mRNA, Prognosis, SOSTDC1

\footnotetext{
*Correspondence: zhaohui3@126.com; mature303@126.com

${ }^{\dagger}$ Equal contributors

${ }^{1}$ Department of Laboratory Medicine, General Hospital of Guangzhou Military

Command of PLA, Guangzhou 510010, Guangdong, China

Full list of author information is available at the end of the article
}

(c) The Author(s). 2018 Open Access This article is distributed under the terms of the Creative Commons Attribution 4.0 International License (http://creativecommons.org/licenses/by/4.0/), which permits unrestricted use, distribution, and reproduction in any medium, provided you give appropriate credit to the original author(s) and the source, provide a link to the Creative Commons license, and indicate if changes were made. The Creative Commons Public Domain Dedication waiver (http://creativecommons.org/publicdomain/zero/1.0/) applies to the data made available in this article, unless otherwise stated. 


\section{Background}

Breast cancer is one of the most commonly diagnosed cancers and one of the leading causes of death among female cancer patients. It has been estimated that, globally, approximately $12 \%$ of the newly diagnosed breast cancers occur in China [1]. Despite great efforts spent on improving the diagnosis and treatment of breast cancer, its prognosis varies greatly among patients. An effective molecular tool is urgently needed for predicting and classifying prognoses of breast cancer patients [2].

Cancer is caused by the accumulation of mutations in cancer susceptibility genes and the resulting abnormal cell growth. In addition to genetic variations, aberrant DNA methylation and variations in gene expression patterns have also been recognised to play an important role in tumourigenesis $[3,4]$. Extensive studies have shown that global DNA hypomethylation and regional hypermethylation of Cytosine-Phosphate-Guanine (CpG) -rich islands are prevalent in cancers [4, 5]. Promoter methylation suppresses gene transcription, and aberrant methylation is one of the major causes leading to instability of the genome, activation of oncogenes and suppression of tumour suppressor genes. Accordingly, aberrant methylation may contribute greatly to breast cancer onset and progression.

Based on variations in gene expression, breast cancer is currently classified into five major subtypes: luminal A, luminal B, ErbB2+, basal-like and normal-like. However, based on the copy number, gene expression and long-term clinical outcomes, breast cancer is further divided into at least 10 intrinsic subtypes, which demonstrate the complexity of the landscape of breast cancer [6]. Each subtype has a unique expression pattern and unique clinical features $[3,7]$ and has a distinct response profile to the same therapy [8]. Thus, attempts to define the prognosis related gene expression signature remain necessary.

Specific methylation profiles may also exist for different subtypes. Holm et al. [9] have reported that certain patterns of hypermethylation, which modulate gene expression and promote tumor progression, may be viable targets in some luminal breast cancers. Reportedly, CpGs in the luminal B subtype are the most frequently methylated and those in the basal-like subtype are the least frequently methylated [10]. Significantly higher methylation levels of tumour suppressor genes Ras Association Domain family 1 (RASSF1) and Glutathione S-transferase Pi 1 (GSTP1) have been observed in the luminal B subtype than in the basal-like subtype [10]. Furthermore, the expression levels of both genes have been shown to be downregulated by hypermethylation in breast cancer [11-15]. The hypermethylation and reduced expression of RASSF1 and GSTP1 have been correlated with cancer onset and progression $[13,14]$.
Despite extensive investigations into aberrant methylation and gene expression, robust and precise molecular prognostic predictors for specific breast cancer subtypes, such as luminal A and B types, remain to be developed. In the present study, we used the data from The Cancer Genome Atlas (TCGA) as a training set and identified methylation sites that are significantly correlated with luminal breast cancer prognosis. The mRNA expression of genes corresponding to these sites correlated significantly with their methylation levels and prognoses. We further compared mRNA expression profiles between breast cancer and normal tissues and identified eight signature genes used for constructing a risk scoring system. Based on this system, luminal breast cancer patients were classified into low-risk and high-risk groups, which exhibited significant prognostic and molecular differences.

\section{Methods}

\section{Data source}

Data on breast cancer methylation and mRNA expression profiles were downloaded from the TCGA data portal (https://gdc-portal.nci.nih.gov/). A total of 1241 samples were available, 628 of which were marked as luminal type (type A or B). Luminal type samples with methylation data (Platform: Illumina Infinium Human Methylation 450) and mRNA-Seq data (Platform: Illumina HiSeq 2000 RNA sequencing) were selected for further analysis. From this analysis, 231 samples were obtained, including 21 control (non-cancerous) tissues and 210 luminal breast cancer tissues. Among the 210 breast cancer tissues, 191 had the corresponding survival information and status.

\section{Primary screening of methylation data}

Continuous variables were expressed as mean \pm standard deviation (SD), and categorical variables were expressed as sample size (composition ratio) in clinical information statistics. Methylation sites with significantly different methylation levels were obtained by comparing the methylation levels between cancer and control samples using the Wilcoxon rank sum test. The influence of the methylation level of these sites on the overall survival of luminal breast cancer patients was analysed using the Cox model. Sites with high correlation were further analysed using a linear correlation model to assess the relationship between their methylation levels and the corresponding mRNA expression. We obtained a subset of methylation sites, the methylation levels of which were significantly correlated with the corresponding mRNA expression. Lastly, the relationship between mRNA expression levels and luminal breast cancer prognosis was assessed using the Cox model. The resulting genes were considered as key genes in luminal breast 
cancer, as their methylation levels and mRNA expression levels were significantly correlated with each other and with the prognosis.

\section{Screening for significantly differentially expressed genes} Based on the cut-off methylation levels (mean methylation levels) of the key genes, samples were classified as hypoor hypermethylated. Differences in expression were analysed by comparing mRNA levels of hypo- and hypermethylation groups with those of the control group (21 samples) using the EdgeR package (R3.1.0) [16]. False discovery rate (FDR) was calculated using the multtest $R$ package. Genes with an FDR of $<0.05$ and an expression fold change of $>1.5$ or $<0.67$ were considered to be significantly differentially expressed genes.

\section{Screening for prognosis-relevant signature and risk score calculation}

Significantly differentially expressed gene mRNAs that significantly correlated with prognosis were screened using Cox regression in the survival $R$ package [17]. $P$-value and the prognosis-relevant coefficient $\beta$ were obtained using log-rank test. Risk score was defined as follows:

$$
\begin{aligned}
\text { Risk score }= & \beta_{\text {gene } 1} \times \text { expr } r_{\text {gene } 1}+\beta_{\text {gene } 2} \times e x p r_{\text {gene } 2}+\cdots \\
& +\beta \tilde{n}^{\text {genen }} \exp r_{\text {genen }},
\end{aligned}
$$

Where $\beta$ and expr are the prognosis-relevant coefficient and expression level of the corresponding gene, respectively. The risk score was calculated for each sample, and the median risk score was set as the cut-off for determining which samples were divided into low-and high-risk groups [18-20].

\section{Correlation analysis between samples and their clinical information}

Clinical information of the corresponding samples, including age, ER status, HER2 status, progesterone receptor (PR) status, pathological stages ( $\mathrm{M}, \mathrm{N}$ and $\mathrm{T})$, radiation therapy and the risk score, were integrated. To identify the clinical features significantly related to prognosis, a prognosis-relevant correlation analysis between the samples and their clinical information was performed using univariate and multivariable Cox regression in the survival $\mathrm{R}$ package. The resulting clinical features were analysed using Kaplan-Meier survival curves.

\section{Validation of the risk scoring system}

To validate the risk scoring system, the expression profile under the accession number GSE22226 (platform GPL1708) [21] from the Gene Expression Omnibus (GEO) database (http://www.ncbi.nlm.nih.gov/gds/?term=) and the Metabric cohort dataset (http://www.cbioportal. org/study?id=brca_metabric\#summary) were downloaded as independent validation datasets. In the GSE22226, a total of 130 breast cancer samples were included in the dataset, 57 of which were of luminal type with survival information and were used for validation. The expression levels of signature genes were extracted, and the risk score was calculated for each sample. The samples were divided into low- and high-risk groups, and the clinical information was also integrated, as described previously. Validation was performed by comparing the distribution of risk scores and overall survival time (in days) as well as by Kaplan-Meier survival curve analysis. In the Metabric cohort, a total of 1979 patients with good follow up data were included, 1140 of which were luminal disease. The risk score was calculated as described previously. Kaplan-Meier survival analysis based on risk score model system and Luminal subtypes were performed. Low- and high-risk groups were divided by signature genes in Luminal A and Luminal B samples from the Metabric cohort and the TCGA database. Prognosis value of low- and high-risk groups were shown by kaplan-Meier survival analysis.

\section{Screening of genes differentially expressed between low- and high-risk groups}

Using the TCGA dataset, the samples were divided into low- and high-risk groups according to their risk scores. Differences in gene expression levels between low- and high-risk groups were analysed using the limma $R$ package, and FDR was calculated using the multtest $R$ package. Genes with an FDR of $<0.05$ were considered to be significantly differentially expressed. A correlation analysis of their expression levels and risk scores was performed, followed by two-way hierarchical clustering (shown as a heat map), Gene Ontology (GO) analysis and KEGG pathway analysis. The entire analysis process is shown in Fig. 1 as a flow chart.

\section{Results}

Features of the samples

A total of 210 luminal breast cancer samples and 21 control samples were included in our analysis, which were obtained from patients with a mean age of $60.73 \pm$ 13.18 and $56.38 \pm 14.59$ years, respectively. Other clinical information, including ER status, can be found in the Supplementary materials (Additional file 1: Figure S1).

Methylation sites associated with breast cancer prognosis We compared the methylation levels of the cancer and control issues using the Wilcoxon rank sum test. A total of 550 methylation sites displayed significant methylation differences (Additional file 2: Figure S2 and Additional file 3: Figure S3). The relationship between methylation levels and breast cancer prognosis was 


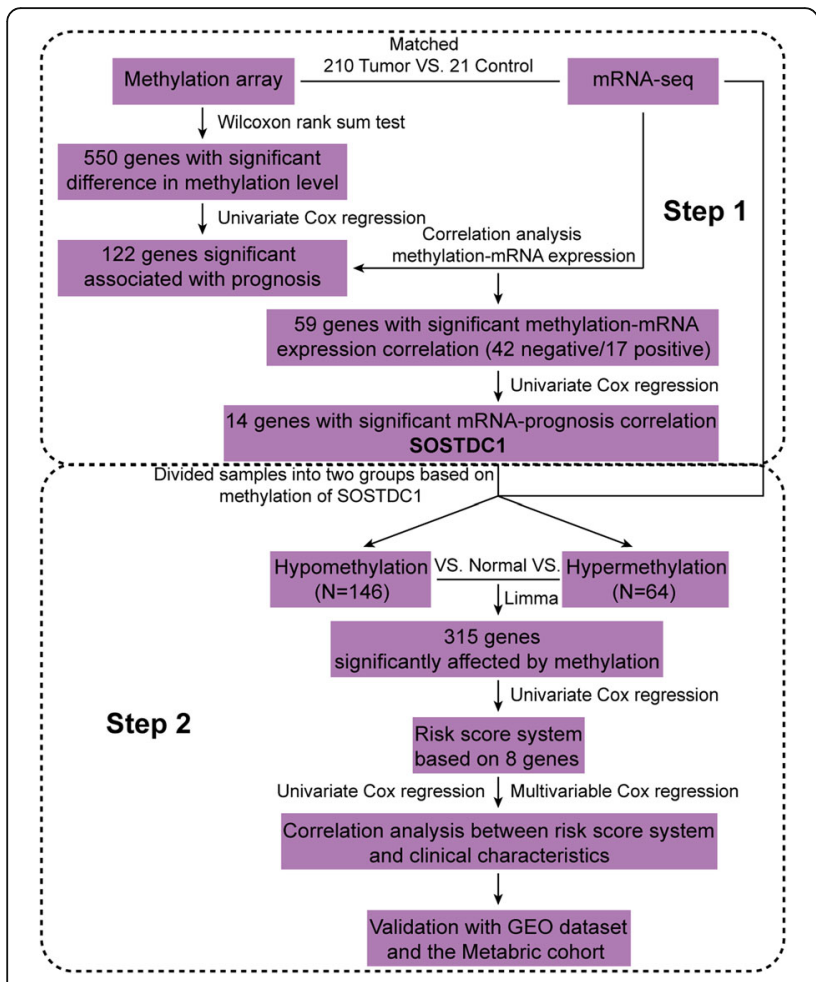

Fig. 1 A flow chart showing the analysis process of this study

analysed using the Cox proportional hazards model, and 122 methylation sites were found to be significantly associated with prognosis (Additional file 4: Figure S4).

Aberrant methylation is considered to be correlated with gene expression. We next evaluated the correlation between methylation levels and mRNA expression. For genes with methylation sites associated with prognosis, their mRNA expression values were extracted from the mRNA-Seq data. The correlation analysis of 122 methylation site-mRNA expression pairs revealed that 59 pairs were significantly correlated $(P<0.05), 42$ of which were negatively correlated and 17 were positively correlated (Additional file 5: Figure S5). Further analysis of the correlation between mRNA expression levels of these genes and prognosis revealed that 14 of them, including VIM, EPHX3, ACVR1, ANGPT1, TPM3, ALOX15, DIO1, KCNJ2, RSPH9, SOSTDC1, SYCP2, MACF1, TDRD5 and CELSR3, were significantly related to breast cancer prognosis (Table 1). As no previous reports showed the existence of the methylation sites of these genes in other types of breast cancer, we consider that the methylation sites of these genes are specific to luminal breast cancer.

\section{Sample grouping based on methylation $\beta$-value of SOSTDC1} One identified gene, sclerostin domain-containing protein 1 (SOSTDC1) was of particular interest, because SOSTDC1 showed a higher methylation level in breast cancer tissues than the other 13 genes (Additional file 6:
Table 1 mRNAs significantly related to luminal breast cancer prognosis

\begin{tabular}{llllll}
\hline Gene & Meth-ID & HR & lower.95 & upper.95 & $p$ \\
\hline VIM & cg12874092 & 0.077 & 0.011 & 0.566 & 0.0117 \\
EPHX3 & cg05488632 & 2.483 & 1.214 & 5.078 & 0.0127 \\
ACVR1 & cg16682903 & 1.120 & 0.675 & 1.351 & 0.0155 \\
ANGPT1 & cg09396217 & 2.472 & 1.116 & 5.474 & 0.0257 \\
TPM3 & cg24490338 & 0.126 & 0.019 & 0.842 & 0.0326 \\
ALOX15 & cg15843823 & 1.419 & 1.018 & 1.976 & 0.0387 \\
DIO1 & cg19526600 & 0.823 & 0.676 & 1.003 & 0.0435 \\
KCNJ2 & cg19042062 & 0.380 & 0.132 & 1.096 & 0.0435 \\
RSPH9 & cg01344171 & 1.481 & 0.962 & 2.282 & 0.0447 \\
SOSTDC1 & cg06363129 & 0.532 & 0.287 & 0.986 & 0.0449 \\
SYCP2 & cg07347645 & 1.628 & 0.969 & 2.737 & 0.0456 \\
MACF1 & cg22233974 & 2.244 & 0.864 & 5.825 & 0.0468 \\
TDRD5 & cg09656934 & 1.414 & 0.951 & 2.104 & 0.0473 \\
CELSR3 & cg06621358 & 0.618 & 0.376 & 1.017 & 0.0481 \\
\hline
\end{tabular}

Table S1) and among the three genes with the highest significant levels (KCNJ2, CELSR3 and SOSTDC1), SOSTDC1 was the only gene that has been reported to be associated with metastatic survival of breast cancer $[22,23]$, which indicates that SOSTDC1 plays a complex role in metastatic breast cancer, so we chose SOSTDC1 for further study.

Extracted data on SOSTDC1 methylation for breast cancer and the control samples indicated that SOSTDC1 methylation levels were significantly higher in cancer tissues than in control tissues $(P=3.05 \mathrm{E}-36)$ (Additional file 7: Table S2). The mean methylation $\beta$-value for breast cancer tissues was determined to be approximately 0.7 (indicated as a black line) and was set as the cut-off. Based on this cut-off value, cancer tissues were divided into hypo- and hypermethylation groups, which contained 64 and 146 samples, respectively.

\section{Screening of differentially expressed genes}

mRNAs with low expression levels (expression value $<5$ ) were removed from TCGA dataset, leaving a total of 11,858 mRNAs. The density peak of the expression levels significantly increased after removal of low-expressing mRNAs (Additional file 8: Table S3). We then compared control samples with both hypo- and hypermethylation samples using significant difference analysis. In total, 217 genes in the SOSTDC1 hypomethylated group and 312 in the SOSTDC1 hypermethylated group displayed significantly differential expression. We obtained 315 genes by combining the two groups. 


\section{Identification of signature genes and construction of the risk scoring system}

The survival information and status of 191 breast cancer tissues in the TCGA dataset were available and used for survival analysis. Among the differentially expressed genes, 67 were identified using Cox regression analysis, with their mRNA levels significantly related to prognosis $(P<0.05)$ (Additional file 9: Table S4).

To identify prognosis-associated signature genes, 67 genes were sorted according to their $P$-values derived from Cox regression log-rank test. Top $n$ genes were used to construct a series of risk scoring systems (Table 2). The corresponding risk scores were calculated, and samples were divided into low- and high-risk groups under each risk scoring system. Their correlation with prognosis and the corresponding area under the curve (AUC) are shown in Table 3. The correlation between low- and high-risk groups and prognosis was at a maximum when the top seven genes were used, while the AUC [20] was maximized when the top eight genes were used. Therefore, we used the top eight genes as signature genes [establishment of cohesion 1 homolog 2 (ESCO2), Protein Kinase $C$ And Casein Kinase Substrate In Neurons 1 (PACSIN1), cell division cycle associated 2 (CDCA2), polymeric immunoglobulin receptor (PIGR), pleiotrophin $(P T N)$, repulsive guidance molecule A $(R G M A)$, kallikrein-related peptidase 4 (KLK4) and centromere protein A (CENPA)].

Risk scores were calculated using the risk scoring system containing the top eight genes (Additional file 10: Table S5). The samples were divided into low- and highrisk groups based on the median risk score (55.27). Kaplan-Meier survival curve analysis showed that sample grouping by this risk scoring system correlated significantly with prognosis (Fig. 2a). Moreover, the overall

Table 2 Prognosis-related genes of luminal breast cancer

\begin{tabular}{llllll}
\hline Symbol & $P$ value $^{\mathrm{a}}$ & Hazard Ratio & $\beta$ & $P$ value $^{\mathrm{b}}$ & AUC \\
\hline ESCO2 & 0.00033 & 1.500 & 5.380 & 0.932 & 0.642 \\
PACSIN1 & 0.00073 & 0.610 & 2.060 & 0.448 & 0.74 \\
CDCA2 & 0.00094 & 2.310 & -7.640 & 0.3137 & 0.781 \\
PIGR & 0.00107 & 0.404 & -1.320 & 0.01191 & 0.878 \\
PTN & 0.00108 & 0.796 & -2.600 & 0.00942 & 0.903 \\
RGMA & 0.0011 & 1.130 & 3.680 & 0.00235 & 0.914 \\
KLK4 & 0.0011 & 0.359 & -1.172 & $0.000251^{c}$ & 0.961 \\
CENPA & 0.00127 & 3.230 & 10.400 & 0.00128 & $0.993^{d}$ \\
ADAMTS14 & 0.00156 & 1.180 & -3.740 & 0.00237 & 0.992 \\
ACAN & 0.00262 & 0.553 & 1.663 & 0.00228 & 0.991 \\
\hline
\end{tabular}

${ }^{a}$ Correlation of gene expression with overall survival

${ }^{\mathrm{b}}$ Correlation of low- and high-risk groups divide by risk score with prognosis

${ }^{c} P$ value with the highest significance

${ }^{\mathrm{d}} P$ value with highest AUC survival was significantly longer in the low-risk group than in the high-risk group $(P=0.00128)$.

We examined the expression levels of the signature genes, which revealed that their expression levels significantly differed between the low- and high-risk groups (Fig. 3a, Additional file 11: Table S6). Significant differences in expression levels were also found between the control samples and the hypo-or hypermethylation groups. (Figure 3b, Additional file 12: Table S7). However, no significant differences were found between the hypo and hypermethylation groups. Moreover, low- and high- risk groups of Luminal A samples divided by the signature genes significantly correlated with the survival ratio. As to Luminal $B$ samples, the survival rate of low risk group was also higher than that of high risk group, although this correlation was not so significant (Fig. 4a). We also evaluated if the prediction analysis of microarray 50 (PAM50) intrinsic subtypes, which are prognostic independent of standard clinicopathologic factors, could well differentiate Luminal A and Luminal B subtypes. As shown in Additional file 13: Table S8, PAM50 was not the key signature genes for splitting Luminal A and Luminal B subtypes in the patient cohort of this study (Additional file 13: Table S8).

To further validate the signature genes, we used the Metabric cohort [6]. The Metabric cohort included 1979 patients with good follow up data of which 1140 were of luminal disease. These luminal samples were also divided into low- and high-risk groups by median risk score based on the signature genes. Low- and high- risk groups of Luminal A samples significantly correlated with the survival ratio. As to Luminal $B$ samples, this correlation also showed no significant difference (Fig. 4b). We combined Luminal A and Luminal B data of the Metabric cohort and made a table $(2 \times 2)$ with Luminal A, Luminal B, high score, low score. We found that the distribution of Luminal A and Luminal B samples between low- and high groups were significantly different. Most Luminal A samples fell in the low score group and the majority of Luminal B samples fell in the high score group (chisq test, $\mathrm{X}$-squared $=137.0685, \quad p<2.2 \mathrm{e}-16) \quad$ (Table 4). KaplanMeier analysis revealed that high score group was significantly correlated with poor survival of patients with luminal breast cancer in Metabric cohort (Additional file 14: Table S9). The classification of Luminal A and Luminal B also significantly correlated with survival ratio of luminal subtype patients. The patients with Luminal A breast cancer had a longer survival time than the patients with Luminal B breast cancer (Additional file 14: Table S9).

\section{Validation of the risk scoring system}

The GSE22226 expression profile dataset was used for validating our risk scoring system. The expression level 
Table 3 Univariate and multivariate Cox regression analysis of the relationship between clinical data and prognosis for TCGA dataset

\begin{tabular}{|c|c|c|c|c|}
\hline \multirow[t]{2}{*}{ Variable } & \multicolumn{2}{|c|}{ Univariate Cox } & \multicolumn{2}{|c|}{ Multivariable Cox } \\
\hline & $p$-value & $\mathrm{HR}(\mathrm{Cl})$ & $p$-value & $\mathrm{HR}(\mathrm{Cl})$ \\
\hline Age $(58.02 \pm 13.28$ y) & 0.163 & $1.045(0.982 \sim 1.11)$ & - & - \\
\hline ER (Positive/Negative) & 0.174 & $0.225(0.0263 \sim 1.929)$ & - & - \\
\hline HER2 (Positive/Negative) & 0.289 & $3.51(0.345 \sim 5.71)$ & - & - \\
\hline pathologic_M(M0/M1) & 0.586 & $1.564(0.331 \sim 2.806)$ & - & - \\
\hline pathologic_N (N0/N1/N2/N3) & 0.639 & $1.159(0.625 \sim 2.15)$ & - & - \\
\hline pathologic_T (T1/T2/T3/T4) & 0.0915 & $1.821(0.909 \sim 2.652)$ & - & - \\
\hline Stage $(I / I / I I I / I V N)$ & 0.121 & $1.586(0.885 \sim 2.841)$ & - & - \\
\hline Radiation therapy (Yes/No) & 0.184 & $0.336(0.0673 \sim 1.679)$ & - & - \\
\hline Luminal type (A/B) & $0.03361^{\mathrm{a}}$ & $1.698(1.036-2.782)$ & $0.0119^{\mathrm{a}}$ & $2.508(1.225 \sim 5.134)$ \\
\hline PR (Positive/Negative) & $0.00713^{\mathrm{a}}$ & $0.125(0.0274 \sim 0.568)$ & 0.4649 & $0.725(0.306 \sim 1.718)$ \\
\hline Riskscore & $0.00115^{\mathrm{a}}$ & $1.095(1.037 \sim 1.156)$ & $0.04382^{\mathrm{a}}$ & $1.229(0.729 \sim 2.071)$ \\
\hline
\end{tabular}

${ }^{\text {a }} P$ values with significance

values of the signature genes were extracted and the risk score for each sample was calculated (Additional file 15: Table S10). Each sample was divided between the low-risk group (29 samples) and high-risk group (28 samples). The survival ratio in both groups was evaluated. Similar to the results seen in the TCGA training dataset, the survival ratio in the low-risk group was significantly higher than that of high-risk group $(P=0.0397)$ in the validation dataset (Fig. 2b). Additionally, the distribution of the risk scores and overall survival time were similar between the validation and training datasets (Fig. $2 \mathrm{c}$ and d). All validation results that we obtained confirmed the robustness and reliability of our risk scoring system.

\section{Correlation between clinical features and prognosis}

Clinical information was integrated for prognosis-related correlation analysis (Additional file 16: Table S11). Univariate Cox regression indicated that both the PR status and the risk score were significantly correlated with prognosis, whereas multivariable Cox regression indicated that only the risk score was an independent prognostic factor (Table 3). Further analysis demonstrated that the survival ratio was higher in the lowrisk group than in the high-risk group in both $\mathrm{PR}$ positive $(\mathrm{PR}+)$ and $\mathrm{PR}$-negative $(\mathrm{PR}-)$ patients (Fig. 5; 14 samples vs. 123 samples; $P=0.105$ vs. $P=0.00552)$. However, the scoring system that we used was more sensitive in $\mathrm{PR}+$ than in $\mathrm{PR}-$ samples because of the difference in $P$-values.

\section{Gene expression differences in the different groups}

We found 121 genes that exhibited significant differential expression $($ FDR $<0.05)$ between the low- and high-risk groups. The correlation analysis indicated that 88 of them were positively correlated and 33 were negatively correlated with the risk score (Additional file 17:
Table S12). The expression patterns of the top 20 positively and negatively correlated genes are shown as heatmaps using hierarchical clustering analysis in Fig. 6a. Further biological function enrichment analysis revealed that most positively correlated genes were involved in the cell cycle, whereas most negatively correlated genes were involved in development, cell adhesion, ion transport and homeostasis (Fig. 6b, Additional file 18: Table S13). KEGG pathway analysis indicated that these genes were correlated with cancer, cell cycle and signalling pathways (Fig. 6c, Additional file 19: Table S14). The overall results of the KEGG pathway analysis were consistent with those of the biological function enrichment analysis, considering the complex relationship between tumourigenesis and multiple biological processes, such as cell cycle, cell adhesion and development.

\section{Discussion}

Variations in methylation profiles are of considerable importance in breast cancer onset and progression [4]. Methylation profiles differ among breast cancer subtypes and may influence gene expression [10]. In the present study, we focused on luminal breast cancer. We downloaded the data from TCGA, a public database that catalogues the genetic profiles of over 30 human tumors, including breast cancer. This platform contains many types of data, such as gene expression, exon expression, miRNA expression, copy number variation (CNV), single nucleotide polymorphism (SNP), mutations, DNA methylation, and protein expression. However, the TCGA database has poor follow up data. A majority of the samples are concatenated shortly after diagnosis, which limited the number of available samples in our study. Due to poor follow up data, the TCGA patient material is not representative of any real breast cancer population. Using data from the TCGA, we identified a 

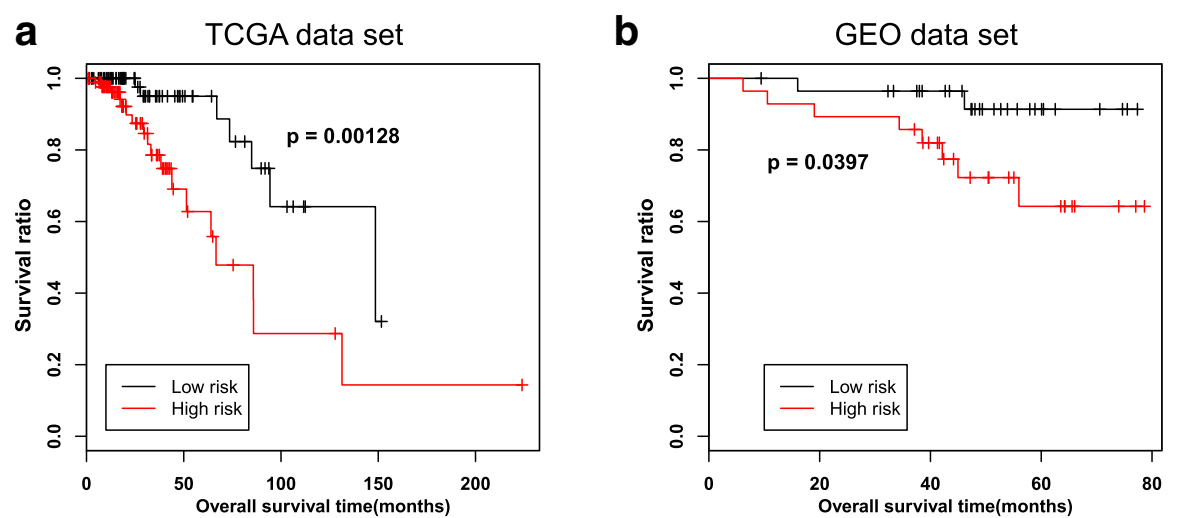

C

TCGA data set

\section{d}
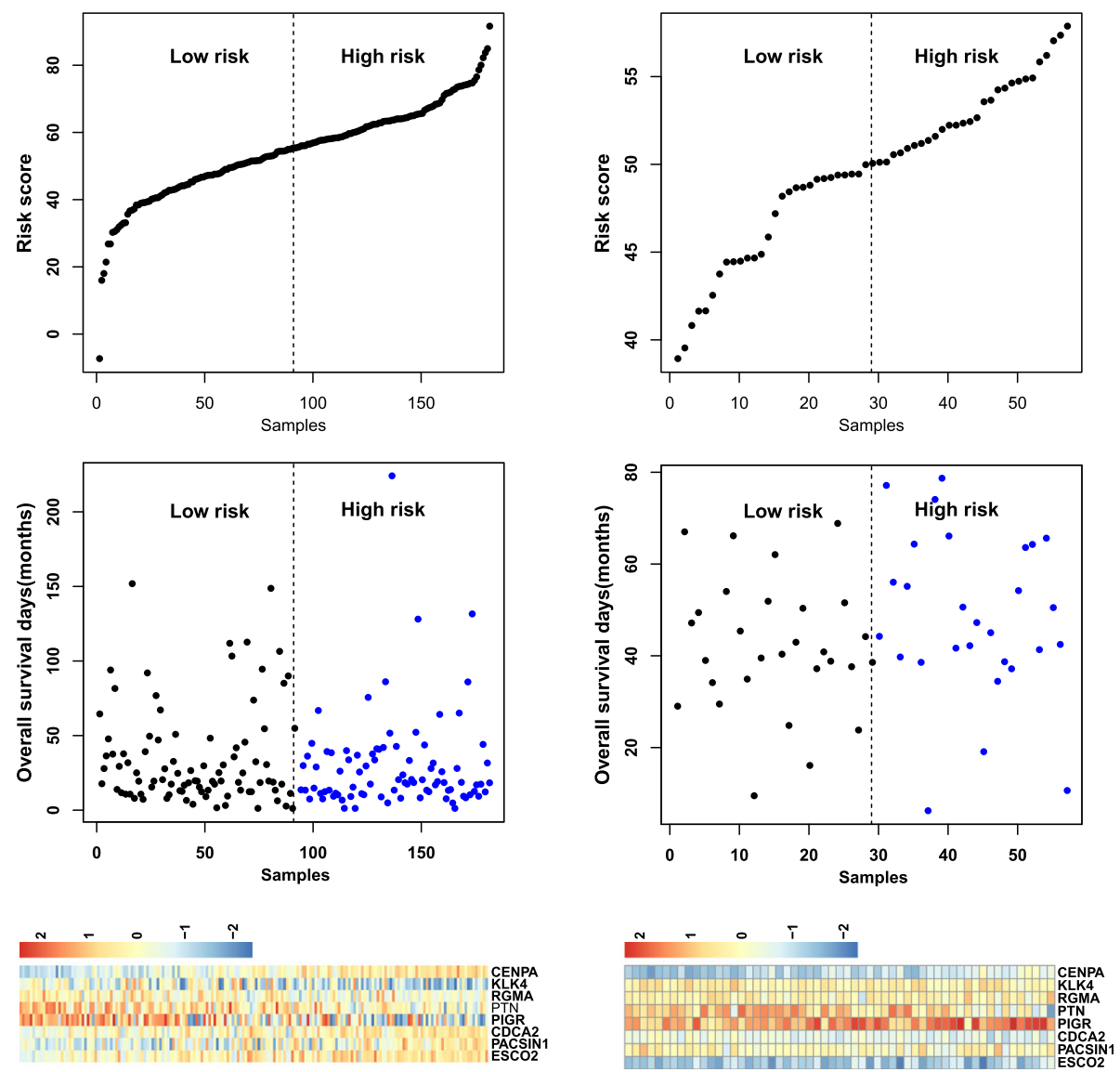

Fig. 2 Comparison of prognosis, risk scores and expression patterns of signature genes. $\mathbf{a}$ and $\mathbf{b}$ Kaplan-Meier survival curves of the low- and high-risk groups between the TCGA and GEO samples. Survival curves of low- and high-risk groups are indicated as black and red lines, respectively. $P$-value indicates significance for the log-rank test. $\mathbf{c}$ and $\mathbf{d}$ Distribution of risk scores, overall survival time and expression profiles of signature genes in the TCGA and GEO samples. Expression profiles are shown as heatmaps

set of prognosis-related methylation sites and further evaluated their relationship with corresponding mRNA expression. We identified 14 genes (Table 2) whose mRNA expression levels, methylation levels and prognosis of breast cancer were significantly correlated.
Among these genes, SOSTDC1 is of special interest, considering its complex role and potential importance in metastatic breast cancer. SOSTDC1 is a member of the sclerostin gene family and is actively involved in the bone morphogenic protein and Wnt signalling pathways. 


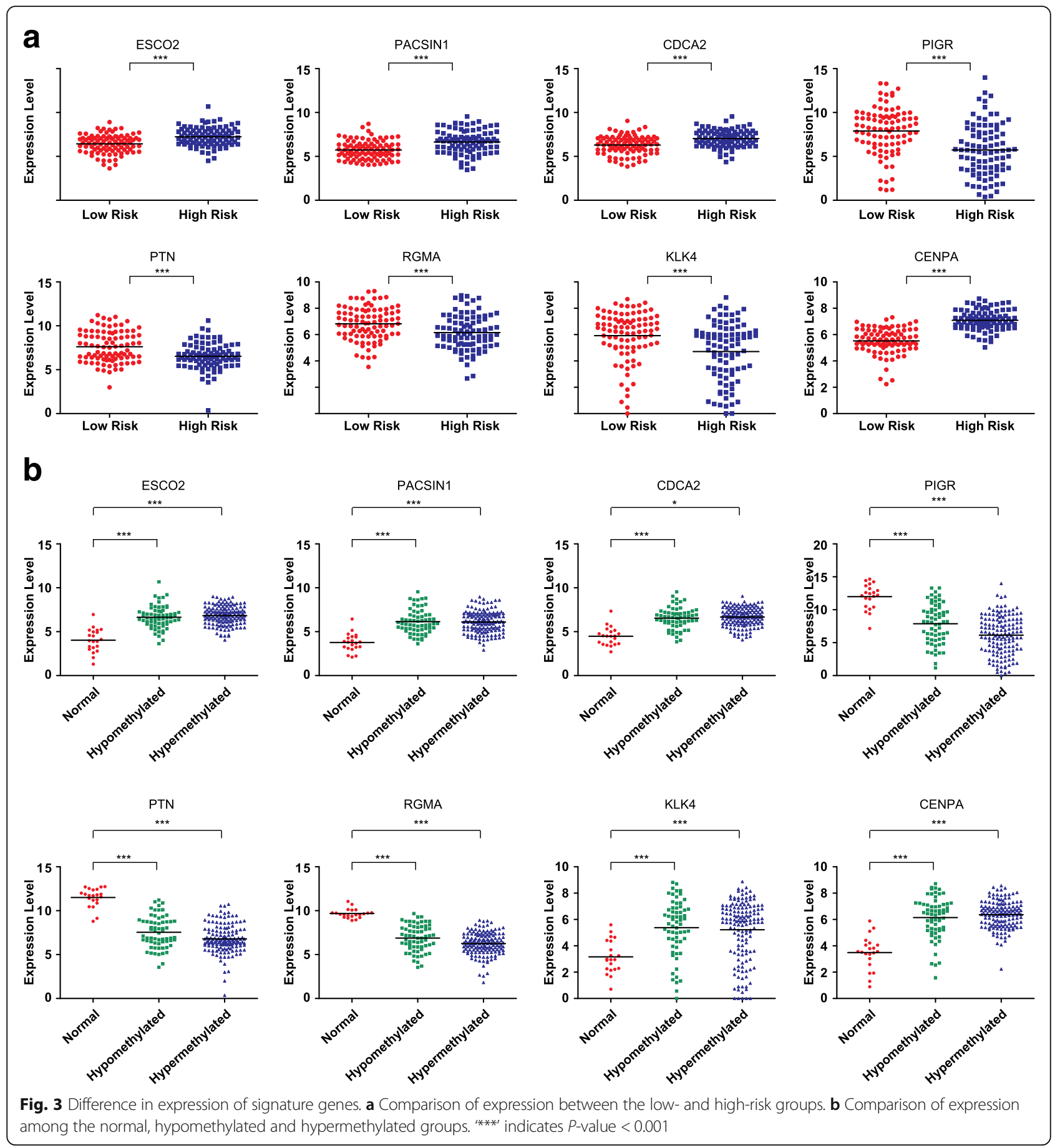

SOSTDC1 mRNA levels are downregulated in breast cancer and are associated with survival $[22,23]$. The elevation in SOSTDC1 methylation level in tumour tissues (Additional file 7: Table S2) may explain SOSTDC1 downregulation in breast cancer because promoter methylation has an inhibitory effect on gene expression. Because SOSTDC1 is closely associated with luminal breast cancer, we divided the samples into hypo- and hypermethylation groups based on
SOSTDC1 methylation levels. Another DNA methylation signature, SAM40, was reported to discriminate patients with luminal A breast cancer between good prognoses and poor prognoses [24]. This highlights the feasibility of the sub-classification of the patient groups based on DNA methylation signature. Future studies might focus on the combination of SAM4O and SOSTDC1 in the prognostic prediction of luminal breast cancer. 


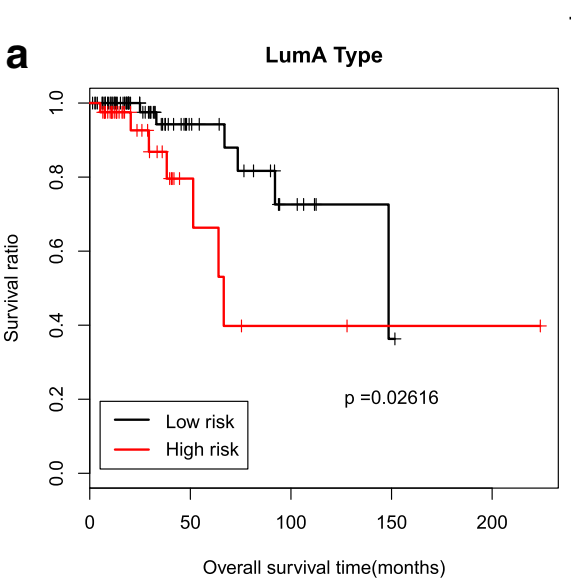

TCGA

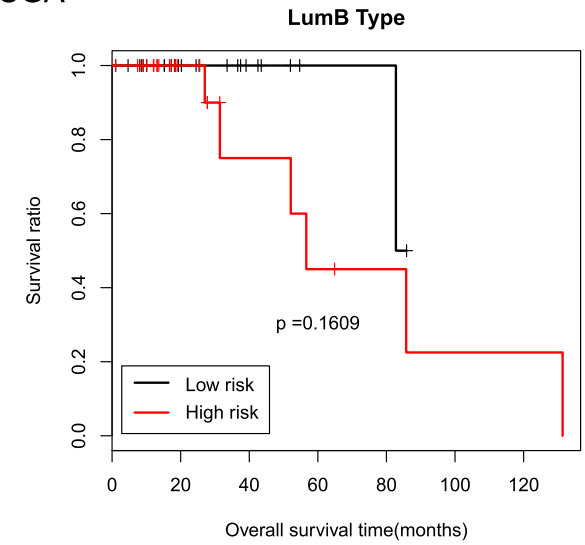

b

BRCA metabric
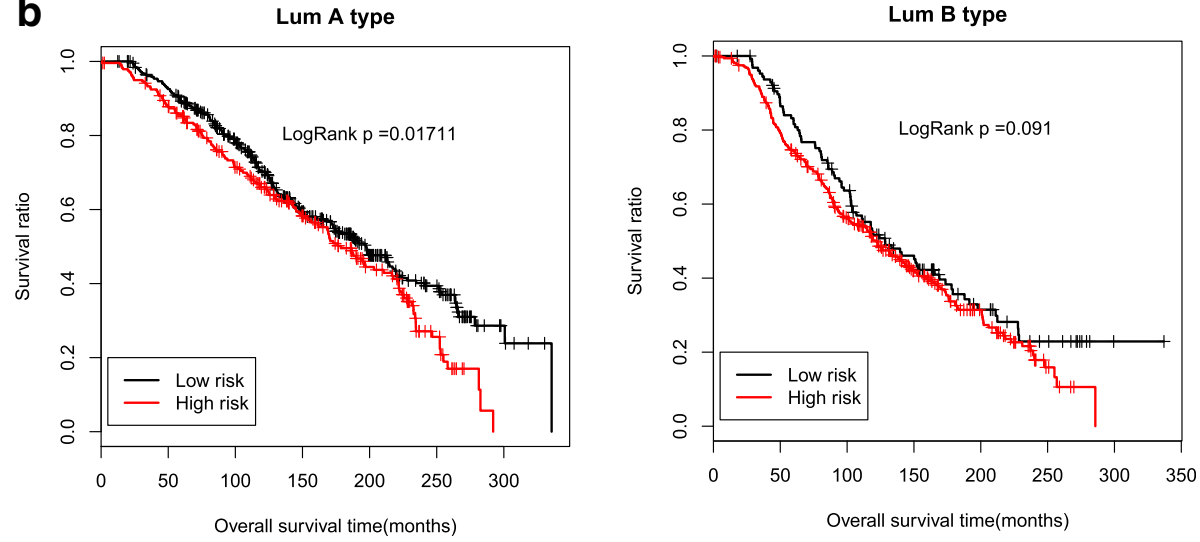

Fig. 4 Prognosis of low- and high-risk groups in Luminal A and Luminal B samples from the TCGA and the Metabric cohort dataset. a KaplanMeier survival curves of low- and high-risk groups divided by eight signature genes in Luminal A and Luminal B samples from the TCGA database, respectively. The black line indicates the low-risk group, and the red line indicates the high-risk group. $\mathbf{b}$ Kaplan-Meier survival curves of low- and high-risk groups divided by eight signature genes in Luminal A and Luminal B samples from the Metabric cohort, respectively. The black line indicates the low-risk group, and the red line indicates the high-risk group

To identify signature genes in luminal breast cancer, we also compared mRNA expression profiles between breast cancer and control tissues. A total of 67 differentially expressed genes were found to be significantly correlated with prognosis. Further analysis identified eight signature genes (ESCO2, PACSIN1, CDCA2, PIGR, PTN, RGMA, $K L K 4$ and CENPA). These signature genes were used to construct a prognosis-related risk scoring system, based on which samples were classified into low-and high-risk groups. The luminal breast cancer samples from the TCGA and the Metabric cohort were used to validate this

Table 4 Fourfold table showing the number of Luminal A and Luminal B samples in low- and high groups

\begin{tabular}{llll}
\hline & Luminal A & Luminal B & $p$ value \\
\hline High group & 242 & 328 & $2.20 \mathrm{E}-16$ \\
Low group & 437 & 133 & \\
\hline
\end{tabular}

system. Interestingly, we found prognostic differences within the Luminal A breast cancer patients in both databases, although the two lines in Fig. $4 \mathrm{~b}$ were almost overlapping. No significant prognostic differences were found within Luminal B samples, indicating that this risk score system might have prognostic value for patients with Luminal A breast cancer.

Many research groups have focused on the prediction of prognosis and chemotherapeutic benefits by construction of a risk system based on gene expression profile, such as the 70-gene predictor [25] and the 50-gene signature [26]. The 50-gene signature test (PAM50) is one of the most widely accepted systems for the prediction of clinical outcomes in women with distinct intrinsic subtypes [26]. In the patient cohorts of this analysis, our signature genes were more suitable for splitting Luminal A and Luminal B subtypes than PAM50. However, a limitation of our study is that the cohort of luminal breast cancer samples in TCGA was small. Future 

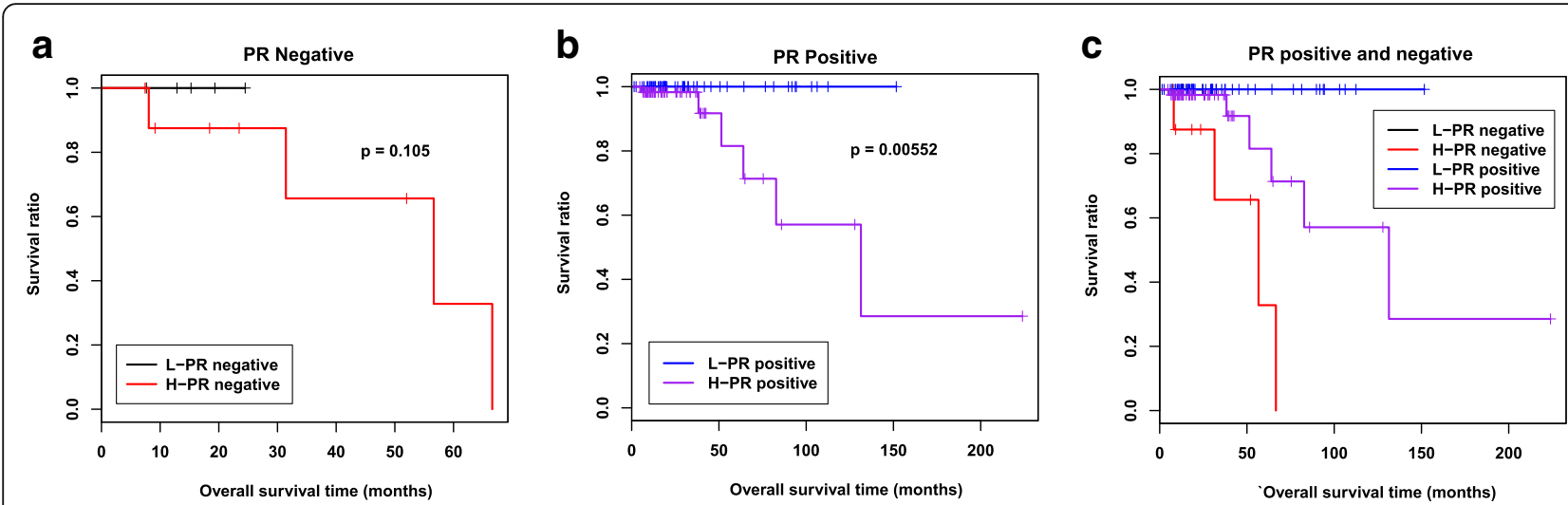

Fig. 5 Prognosis of the low- and high-risk groups in PR-negative and PR-positive samples. a Kaplan-Meier survival curves of the low-and high-risk groups in PR-negative samples. The black line indicates the low-risk group, and the red line indicates the high-risk group. P-value indicates the significance of the difference between the two groups. b Kaplan-Meier survival curves of the low- and high-risk groups in PR-positive samples. The blue line indicates the low-risk group, and the violet line indicates the high-risk group. P-value indicates the significance of the difference between the two groups. c Combination of the Kaplan-Meier survival curves from (a) and (b)

studies will utilize larger patient cohorts and enrich the clinical data to validate our risk system.

Previous studies have shown that most signature genes are involved in cancer progression, even though they may not be directly involved in breast cancer. It has been reported that ESCO2, CDCA2 and CENPA are cell cycle-related genes involved in cancer progression. ESCO2 is an acetyltransferase, which is required for cohesion acetylation and the establishment of sister chromatid cohesion in the $S$ phase $[27,28]$, and has been found to be upregulated in melanoma [29]. CDCA2 is required in the formation of mitotic chromatin and is involved in the progression of human squamous cell carcinoma [30]. CENPA is essential for centromere integrity and chromosome segregation, and CENPA dysregulation may promote tumourigenesis due to the resulting genome instability [31-33]. Other signature genes, including PTN, KLK4, RGMA and PIGR, have also been reported to be involved in cancer progression. Increased PTN [34, 35] and KLK4 [36-38] expression is strongly

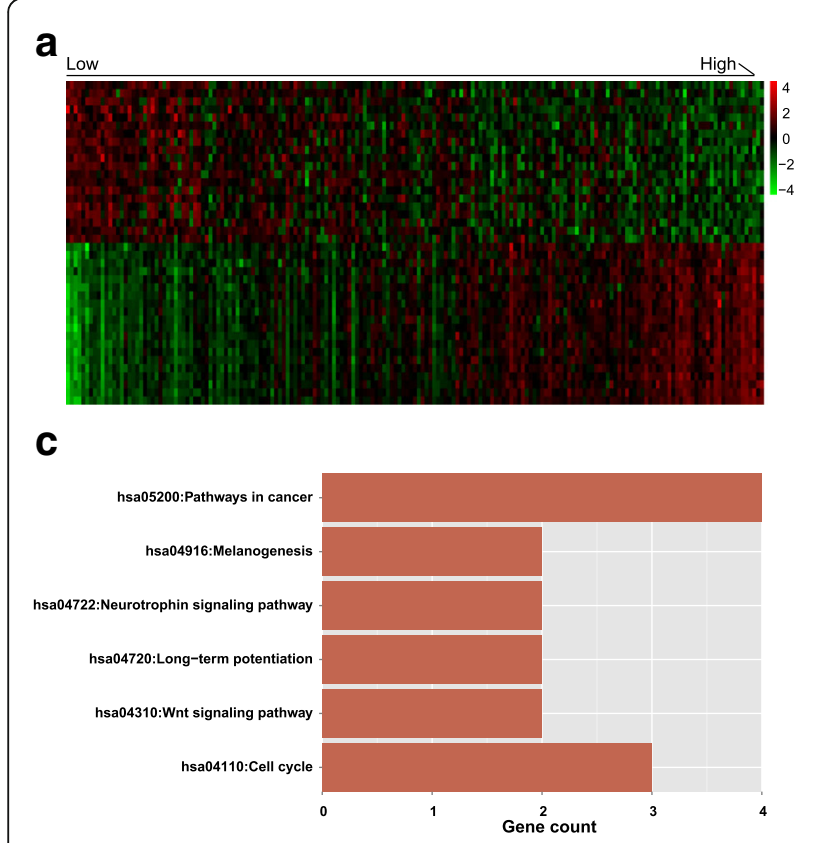

b

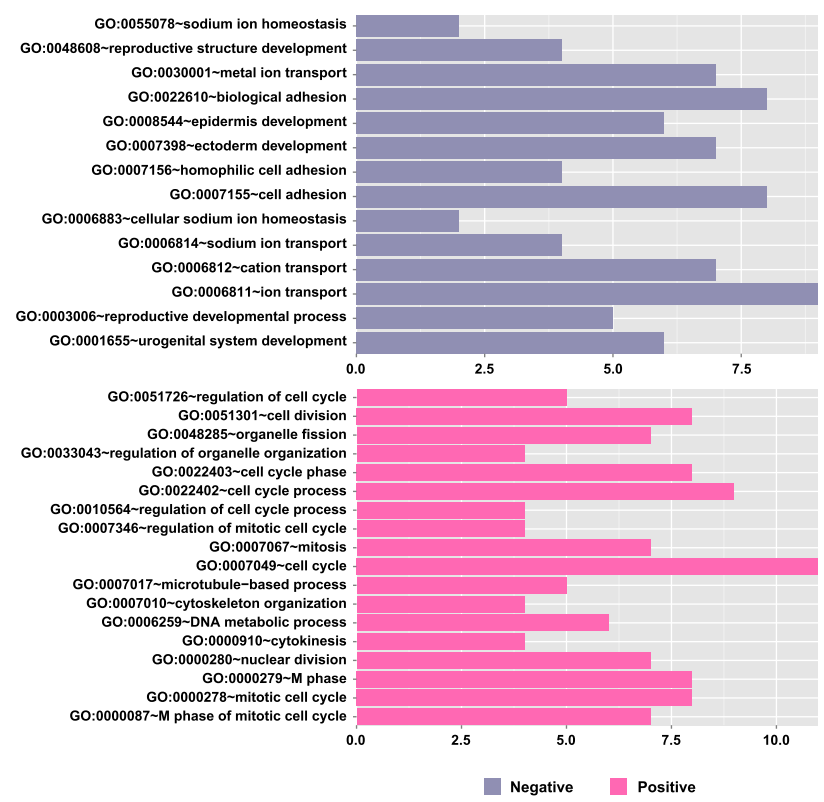

Fig. 6 Functional annotation of genes differentially expressed between the low- and high-risk groups. a Hierarchical clustering analysis of the expression levels of the top 20 positively and negatively related genes. $\mathbf{b} \mathrm{GO}$ analysis of negatively (upper) and positively (lower) related genes. c KEGG pathway analysis of significantly correlated genes 
associated with the progression of different malignant cancers. Decreased PIGR expression has been found in colon tumours [39], while $R G M A$ has been reported to have an inhibitory effect on cancer progression [40, 41]. The remaining signature gene, PACSIN1, is important in endocytosis and synaptic vesicle recycling [42, 43]. Although its direct involvement in cancer has not been reported, it may play an indirect role in cancer progression.

Our results also demonstrated significant differences in the expression of these signature genes between lowand high-risk groups and between the control and cancerous tissues (Fig. 3). Our GO and pathway analyses revealed that the genes that were expressed differentially between the low- and high-risk groups were mainly involved in biological processes, such as cell cycle and cancer progression (Fig. 5b and c).

There are limitations in our manuscript. The gene signature is derived from the segregation of patients based on methylation level of only one gene (SOSTDC1), which could cause bias of data analysis. The eight-gene signature was screened based on bioinformatics analysis and this study may just provide clues for future study of patients with luminal breast cancer. The future focus of our work is to collect more samples and improve our risk score system experimentally.

Taken together, our results supported the role of these genes, consistent with their biological functions, in the development and progression of luminal breast cancer.

\section{Conclusions}

In conclusion, we identified 14 genes that were closely related to luminal breast cancer prognosis. Their methylation levels, mRNA expression and prognosis were significantly correlated with each other. We also identified eight clinically valuable signature genes for luminal breast cancer, and a risk scoring system was built upon this profile. Our results demonstrated that this system is stable and effective in predicting prognosis and can be used in the clinical diagnosis and treatment of luminal breast cancer. Further functional studies on the signature genes are needed to gain a deeper insight into the roles they play in breast cancer onset, with specific emphasis on PACSIN1 because its direct involvement in cancer has not yet been reported.

\section{Additional files}

Additional file 1: Figure S1. Box plot showing the methylation levels of 14 genes in the control and luminal breast cancer tissues. Blue and orange boxes indicate methylation levels in the control and cancer samples, respectively. (PDF $20 \mathrm{~kb}$ )

Additional file 2: Figure S2. Distribution of SOSTDC1 methylation levels in the control and luminal breast cancer tissues. Red and blue points indicate methylation levels in the control and cancer samples, respectively. Black lines indicate the mean methylation levels of the corresponding samples. (PDF $19 \mathrm{~kb}$ )

Additional file 3: Figure S3. Distribution of mRNA expression density. Solid and dashed lines indicate the density distribution curve before and after the removal of mRNA with low expression levels, respectively. (PDF $7 \mathrm{~kb}$ )

Additional file 4: Figure S4. Kaplan-Meier survival curves of low- and high-risk groups divided by PAM50 in Luminal A and Luminal B samples, respectively. The black line indicates the low-risk group, and the red line indicates the high-risk group. (TIFF $772 \mathrm{~kb}$ )

Additional file 5: Figure S5. Kaplan-Meier survival analysis based on risk score model system (a) and Luminal subtypes using the Metabric cohort (b). (a) The black and red lines indicates the low-risk group and the high-risk group; (b) The black and red lines indicates the Luminal A and Luminal B breast cancer tissues. (PDF $29 \mathrm{~kb}$ )

Additional file 6: Table S1. Clinical information of 210 luminal breast cancer samples. (XLSX $10 \mathrm{~kb}$ )

Additional file 7: Table S2. List of DEmethylated sites. (XLS $105 \mathrm{~kb}$ )

Additional file 8: Table S3. Intersection of DEGs in hypo and hyper methylated groups. (XLS $96 \mathrm{~kb}$ )

Additional file 9: Table S4. Methylation sites that significantly associated with breast cancer prognosis. (XLS $38 \mathrm{~kb}$ )

Additional file 10: Table S5. Correlated methylation site-mRNA expression pairs. (XLS $24 \mathrm{~kb}$ )

Additional file 11: Table S6. Survival prognostis related genes from cox regression. (XLS $22 \mathrm{~kb}$ )

Additional file 12: Table S7. Clinic and gene factor information for model. (XLS $62 \mathrm{~kb}$ )

Additional file 13: Table S8. Model gene factor expression in Low and High risk samples. (XLS $52 \mathrm{~kb}$ )

Additional file 14: Table S9. Model gene factor expression in Normal and Cancer samples. (XLS $57 \mathrm{~kb}$ )

Additional file 15: Table S10. Clinic and gene expression information for testing dataset GSE22226. (XLS $32 \mathrm{~kb}$ )

Additional file 16: Table S11. Clinic information for samples in this study. (XLS $56 \mathrm{~kb}$ )

Additional file 17: Table S12. DEGs screened from High and low risk groups. (XLS $31 \mathrm{~kb}$ )

Additional file 18: Table S13-1. Biology process annotations for DEGs negatively correlated to High and Low risk. Table S13-2. Biology process annotations for DEGs positively correlated to High and Low risk. (XLS $23 \mathrm{~kb}$ )

Additional file 19: Table S14. KEGG pathways annotations for DEGs from High and Low risk. (XLSX $10 \mathrm{~kb}$ )

\section{Abbreviations}

AUC: area under the curve; CDCA2: cell division cycle associated 2; CENPA: centromere protein $\mathrm{A}$; CNV: copy number variation; CpG: Cytosine-Phosphate-Guanine; ESCO2: establishment of cohesion 1 homolog 2; GEO: Gene Expression Omnibus; GO: Gene Ontology; GSTP1: Glutathione S-transferase Pi 1; KLK4: kallikrein-related peptidase 4; PACSIN1: Protein Kinase C And Casein Kinase Substrate In Neurons 1; PAM50: the prediction analysis of microarray 50; PIGR: polymeric immunoglobulin receptor; PR: progesterone receptor; PTN: pleiotrophin; RASSF1: Ras Association Domain family 1; RGMA: repulsive guidance molecule A; SD: standard deviation; SNP: single nucleotide polymorphism; SOSTDC1: sclerostin domain-containing protein 1; TCGA: The Cancer Genome Atlas

\section{Acknowledgements}

Not applicable.

Funding

Data collection and Data analysis of this work were supported by The Military logistics Research Project (number CWH17C017 to Linhai Li), Science 
and Technology Program of Guangzhou, China (number 201804010186 to Bin Xiao) and The Natural Science Foundation of China (number 31672536 to Wenbo Hao)

\section{Availability of data and materials}

The dataset supporting the conclusions of this article is included within the article and its supplemental tables. Methylation and mRNA expression of breast cancer tissues were mined from The Cancer Genome Atlas (TCGA) (https://cancergenome.nih.gov/).

\section{Authors' contributions}

LHL and ZHS conceived and designed the study. BX, LDC, YLK, JFH, LC, RZ, WYZ and JYC contributed to the computational analyses and confirmed the results. $\mathrm{LL}$ and $\mathrm{WBH}$ gave advice on the software applications. All authors have read and approved the final version of this manuscript.

\section{Ethics approval and consent to participate}

The data of breast cancer patients we used in this study are all from the publicly accessible TCGA database. Separate ethics committee approval is not required.

\section{Consent for publication}

Not applicable.

\section{Competing interests}

The authors declare that they have no competing interests.

\section{Publisher's Note}

Springer Nature remains neutral with regard to jurisdictional claims in published maps and institutional affiliations.

\section{Author details}

'Department of Laboratory Medicine, General Hospital of Guangzhou Military Command of PLA, Guangzhou 510010, Guangdong, China. ${ }^{2}$ Department of Breast Surgery, General Hospital of Guangzhou Military Command of PLA Guangzhou 510010, Guangdong, China. Institute of Antibody Engineering, School of Biotechnology, Southern Medical University, Guangzhou, China. ${ }^{4}$ State Key Laboratory of Organ Failure, Institute of Antibody Engineering, School of Biotechnology, Southern Medical University, Guangzhou, China. ${ }^{5}$ Guangdong Provincial Key Laboratory of Tropical Disease Research, Schoo of Public Health, Southern Medical University, Guangzhou, China.

\section{Received: 20 July 2017 Accepted: 26 March 2018}

\section{Published online: 11 April 2018}

\section{References}

1. Fan L, Strasser-Weippl K, Li J-J, St Louis J, Finkelstein D, Yu K-D, Chen W-Q, Shao Z-M, Goss P. Breast cancer in China. The lancet oncology. 2014;15(7):e279-89.

2. Brenton J, Carey L, Ahmed A, Caldas C. Molecular classification and molecular forecasting of breast cancer: ready for clinical application? J Clin Oncol. 2005;23(29):7350-60.

3. Perou C, Sorlie T, Eisen M, van de Rijn M, Jeffrey S, Rees C, Pollack J, Ross D, Johnsen $\mathrm{H}$, Akslen $\mathrm{L}$, et al. Molecular portraits of human breast tumours. Nature. 2000:406(6797):747-52.

4. Karsli-Ceppioglu S, Dagdemir A, Judes G, Ngollo M, Penault-Llorca F, Pajon A, Bignon Y, Bernard-Gallon D. Epigenetic mechanisms of breast cancer: an update of the current knowledge. Epigenomics. 2014;6(6):651-64.

5. Jaenisch R, Bird A. Epigenetic regulation of gene expression: how the genome integrates intrinsic and environmental signals. Nat Genet. 2003:33(Suppl):245-54.

6. Curtis C, Shah S, Chin S, Turashvili G, Rueda O, Dunning M, Speed D, Lynch A, Samarajiwa $S$, Yuan $Y$, et al. The genomic and transcriptomic architecture of 2,000 breast tumours reveals novel subgroups. Nature. 2012;486(7403):346-52.

7. Sorlie T, Perou C, Tibshirani R, Aas T, Geisler S, Johnsen H, Hastie T, Eisen M, van de Rijn M, Jeffrey S, et al. Gene expression patterns of breast carcinomas distinguish tumor subclasses with clinical implications. Proc Natl Acad Sci U S A. 2001;98(19):10869-74.

8. Ades F, Zardavas D, Bozovic-Spasojevic I, Pugliano L, Fumagalli D, de Azambuja E, Viale G, Sotiriou C, Piccart M. Luminal B breast cancer: molecular characterization, clinical management, and future perspectives. J Clin Oncol. 2014:32(25):2794-803.

9. Holm K, Staaf J, Lauss M, Aine M, Lindgren D, Bendahl P, Vallon-Christersson J, Barkardottir R, Hoglund M, Borg A, et al. An integrated genomics analysis of epigenetic subtypes in human breast tumors links DNA methylation patterns to chromatin states in normal mammary cells. Breast Cancer Res. 2016;18(1):27

10. Holm K, Hegardt C, Staaf J, Vallon-Christersson J, Jonsson G, Olsson H, Borg A, Ringner M. Molecular subtypes of breast cancer are associated with characteristic DNA methylation patterns. Breast Cancer Res. 2010; 12(3):R36.

11. Burbee D, Forgacs E, Zochbauer-Muller S, Shivakumar L, Fong K, Gao B, Randle D, Kondo M, Virmani A, Bader S, et al. Epigenetic inactivation of RASSF1A in lung and breast cancers and malignant phenotype suppression. J Natl Cancer Inst. 2001;93(9):691-9.

12. Dammann R, Schagdarsurengin U, Seidel C, Strunnikova M, Rastetter M, Baier K, Pfeifer G. The tumor suppressor RASSF1A in human carcinogenesis: an update. Histol Histopathol. 2005;20(2):645-63.

13. Alvarez C, Tapia T, Cornejo V, Fernandez W, Munoz A, Camus M, Alvarez M, Devoto L, Carvallo P. Silencing of tumor suppressor genes RASSF1A, SLIT2, and WIF1 by promoter hypermethylation in hereditary breast cancer. Mol Carcinog. 2013;52(6):475-87.

14. Lasabova Z, Tilandyova P, Kajo K, Zubor P, Burjanivova T, Danko J, Plank L. Hypermethylation of the GSTP1 promoter region in breast cancer is associated with prognostic clinicopathological parameters. Neoplasma. 2010;57(1):35-40.

15. Louie S, Grossman E, Crawford L, Ding L, Camarda R, Huffman T, Miyamoto D, Goga A, Weerapana E, Nomura D. GSTP1 is a driver of triple-negative breast Cancer cell metabolism and pathogenicity. Cell chemical biology. 2016:23(5):567-78

16. Ritchie M, Phipson B, Wu D, Hu Y, Law C, Shi W, Smyth G. Limma powers differential expression analyses for RNA-sequencing and microarray studies. Nucleic Acids Res. 2015;43(7):e47.

17. Singh R, Mukhopadhyay K. Survival analysis in clinical trials: basics and must know areas. Perspect Clin Res. 2011;2(4):145-8.

18. Bao Z, Li M, Wang J, Zhang C, Wang H, Yan W, Liu Y, Zhang W, Chen L, Jiang $T$. Prognostic value of a nine-gene signature in glioma patients based on mRNA expression profiling. CNS Neurosci Ther. 2014;20(2):112-8.

19. Cheng W, Ren X, Cai J, Zhang C, Li M, Wang K, Liu Y, Han S, Wu A. A fivemiRNA signature with prognostic and predictive value for MGMT promotermethylated glioblastoma patients. Oncotarget. 2015;6(30):29285-95.

20. Zhang C, Zhu P, Yang P, Cai J, Wang Z, Li Q, Bao Z, Zhang W, Jiang T. Identification of high risk anaplastic gliomas by a diagnostic and prognostic signature derived from mRNA expression profiling. Oncotarget. 2015;6(34):36643-51.

21. Esserman L, Berry D, Cheang M, Yau C, Perou C, Carey L, Demichele A, Gray J, Conway-Dorsey $\mathrm{K}$, Lenburg $\mathrm{M}$, et al. Chemotherapy response and recurrence-free survival in neoadjuvant breast cancer depends on biomarker profiles: results from the I-SPY 1 TRIAL (CALGB 150007/150012; ACRIN 6657). Breast Cancer Res Treat. 2012:132(3):1049-62.

22. Clausen K, Blish K, Birse C, Triplette M, Kute T, Russell G, D'Agostino RB Jr, Miller L, Torti F, Torti S. SOSTDC1 differentially modulates Smad and beta-catenin activation and is down-regulated in breast cancer. Breast Cancer Res Treat. 2011;129(3):737-46.

23. Rawat A, Gopisetty G, Thangarajan R. E4BP4 is a repressor of epigenetically regulated SOSTDC1 expression in breast cancer cells. Cell Oncol (Dordr) 2014:37(6):409-19.

24. Fleischer T, Klajic J, Aure M, Louhimo R, Pladsen A, Ottestad L, Touleimat N, Laakso M, Halvorsen A, Grenaker A, et al. DNA methylation signature (SAM40) identifies subgroups of the luminal a breast cancer samples with distinct survival. Oncotarget. 2017;8(1):1074-82.

25. Cardoso F, Van'T VL, Bogaerts J, Slaets L, Viale G, Delaloge S, Pierga JY, Brain E, Causeret S, DeLorenzi M, et al. 70-gene signature as an aid to treatment decisions in early-stage breast Cancer. N Engl J Med. 2016:375(8):717-29.

26. Parker J, Mullins $M$, Cheang $M$, Leung $S$, Voduc $D$, Vickery $T$, Davies $S$, Fauron $\mathrm{C}, \mathrm{He} X, \mathrm{Hu}$ Z, et al. Supervised risk predictor of breast cancer based on intrinsic subtypes. J Clin Oncol. 2009;27(8):1160-7.

27. Whelan G, Kreidl E, Wutz G, Egner A, Peters J, Eichele G. Cohesin acetyltransferase Esco2 is a cell viability factor and is required for cohesion in pericentric heterochromatin. EMBO J. 2012;31(1):71-82. 
28. Kim B, Kang K, Jung S, Choi H, Seo J, Chae J, Cho E, Youn H, Qin J, Kim S. Esco2 is a novel corepressor that associates with various chromatin modifying enzymes. Biochem Biophys Res Commun. 2008;372(2):298-304.

29. Ryu B, Kim D, Deluca A, Alani R. Comprehensive expression profiling of tumor cell lines identifies molecular signatures of melanoma progression. PLoS One. 2007;2(7):e594.

30. Uchida F, Uzawa K, Kasamatsu A, Takatori H, Sakamoto Y, Ogawara K, Shiiba M, Bukawa H, Tanzawa H. Overexpression of CDCA2 in human squamous cell carcinoma: correlation with prevention of G1 phase arrest and apoptosis. PLoS One. 2013;8(2):e56381.

31. Giunta S, Funabiki H. Integrity of the human centromere DNA repeats is protected by CENP-A, CENP-C, and CENP-T. Proc Natl Acad Sci U S A. 2017;114(8):1928-33.

32. Tomonaga T, Matsushita K, Yamaguchi S, Oohashi T, Shimada H, Ochiai T, Yoda K, Nomura F. Overexpression and mistargeting of centromere proteina in human primary colorectal cancer. Cancer Res. 2003;63(13):3511-6.

33. Athwal R, Walkiewicz M, Baek S, Fu S, Bui M, Camps J, Ried T, Sung M, Dalal Y. CENP-A nucleosomes localize to transcription factor hotspots and subtelomeric sites in human cancer cells. Epigenetics Chromatin. 2015;8:2.

34. Zhang $N$, Zhong $R$, Wang Z, Deuel T. Human breast cancer growth inhibited in vivo by a dominant negative pleiotrophin mutant. J Biol Chem. 1997:272(27):16733-6.

35. Kadomatsu K, Muramatsu T. Midkine and pleiotrophin in neural development and cancer. Cancer Lett. 2004;204(2):127-43.

36. Ramsay A, Dong Y, Hunt M, Linn M, Samaratunga H, Clements J, Hooper J. Kallikrein-related peptidase 4 (KLK4) initiates intracellular signaling via protease-activated receptors (PARS). KLK4 and PAR-2 are co-expressed during prostate cancer progression. J Biol Chem. 2008;283(18):12293-304.

37. Kontos C, Chantzis D, Papadopoulos I, Scorilas A. Kallikrein-related peptidase 4 (KLK4) mRNA predicts short-term relapse in colorectal adenocarcinoma patients. Cancer Lett. 2013;330(1):106-12.

38. Papagerakis $P$, Pannone $G$, Zheng $L$, Athanassiou-Papaefthymiou M, Yamakoshi Y, Mcguff H, Shkeir O, Ghirtis K, Papagerakis S. Clinical significance of kallikrein-related peptidase-4 in oral cancer. Anticancer Res. 2015;35(4):1861-6

39. Traicoff J, De Marchis L, Ginsburg B, Zamora R, Khattar N, Blanch V, Plummer S, Bargo S, Templeton D, Casey G, et al. Characterization of the human polymeric immunoglobulin receptor (PIGR) 3'UTR and differential expression of PIGR mRNA during colon tumorigenesis. J Biomed Sci. 2003;10(6 Pt 2): 792-804.

40. Li J, Ye L, Kynaston H, Jiang W. Repulsive guidance molecules, novel bone morphogenetic protein co-receptors, are key regulators of the growth and aggressiveness of prostate cancer cells. Int J Oncol. 2012;40(2):544-50.

41. Li J, Ye L, Sanders A, Jiang W. Repulsive guidance molecule B (RGMB) plays negative roles in breast cancer by coordinating BMP signaling. J Cell Biochem. 2012;113(7):2523-31.

42. Andersson F, Jakobsson J, Low P, Shupliakov O, Brodin L. Perturbation of syndapin/PACSIN impairs synaptic vesicle recycling evoked by intense stimulation. J Neurosci. 2008;28(15):3925-33.

43. Qualmann B, Roos J, Digregorio P, Kelly R. Syndapin I, a synaptic dynaminbinding protein that associates with the neural Wiskott-Aldrich syndrome protein. Mol Biol Cell. 1999;10(2):501-13.

\section{Submit your next manuscript to BioMed Central and we will help you at every step:}

- We accept pre-submission inquiries

- Our selector tool helps you to find the most relevant journal

- We provide round the clock customer support

- Convenient online submission

- Thorough peer review

- Inclusion in PubMed and all major indexing services

- Maximum visibility for your research

Submit your manuscript at www.biomedcentral.com/submit

) Biomed Central 